\title{
The knowns and unknowns of latent Mycobacterium tuberculosis infection
}

\author{
W. Henry Boom, ${ }^{1,2,3}$ Ulrich E. Schaible, ${ }^{4,5}$ and Jacqueline M. Achkar ${ }^{6,7}$ \\ DDepartment of Medicine, '²epartment of Pathology, and ${ }^{3}$ Department of Molecular Biology and Microbiology, Case Western Reserve University and University Hospitals Cleveland Medical Center, Cleveland, \\ Ohio, USA. ${ }^{4}$ Division of Cellular Microbiology, Research Center Borstel-Leibniz Lung Center, Borstel, Germany. ${ }^{5}$ German Center for Infection Research, partner site Hamburg-Lübeck-Borstel-Riems, Cermany. \\ ${ }^{6}$ Department of Medicine and ${ }^{7}$ Department of Microbiology and Immunology, Albert Einstein College of Medicine, Bronx, New York, USA.
}

\begin{abstract}
Humans have been infected with Mycobacterium tuberculosis (Mtb) for thousands of years. While tuberculosis (TB), one of the deadliest infectious diseases, is caused by uncontrolled Mtb infection, over $90 \%$ of presumed infected individuals remain asymptomatic and contain Mtb in a latent TB infection (LTBI) without ever developing disease, and some may clear the infection. A small number of heavily Mtb-exposed individuals appear to resist developing traditional LTBI. Because Mtb has mechanisms for intracellular survival and immune evasion, successful control involves all of the arms of the immune system. Here, we focus on immune responses to Mtb in humans and nonhuman primates and discuss new concepts and outline major knowledge gaps in our understanding of LTBI, ranging from the earliest events of exposure and infection to success or failure of Mtb control.
\end{abstract}

\section{Introduction}

Mycobacterium tuberculosis (Mtb), a bacterium transmitted through respiratory droplets, is one of the most successful human pathogens. With approximately 10 million cases and 1.45 million associated deaths per year, tuberculosis (TB), which is caused by uncontrolled Mtb infection, is the world's most lethal infectious disease next to COVID-19 (1). Failure of TB control programs and the lack of a highly efficacious vaccine against TB have refocused attention on the earliest events in TB pathogenesis - the acquisition and control of Mtb bacilli in the human lung. Because of its ability to infect and survive in macrophages (reviewed in ref. 2), Mtb can persist and cause, in most individuals, a clinically inapparent infection referred to as latent TB infection (LTBI) (reviewed in ref. 3). However, TB and LTBI are not binary classifications but rather terms comprising a heterogeneous spectrum (reviewed in ref. 4). Our inability to detect persistent/latent Mtb bacilli makes it impossible to determine who among those presumed infected and asymptomatic have cleared the bacilli (5), remain latently infected, or will progress to uncontrolled infection/TB (Table 1). Instead, we rely on a detectable cellular immune response to $\mathrm{Mtb}$ antigens in the form of a positive tuberculin skin test (TST) and/ or blood-based IFN- $\gamma$ release assay (IGRA) as surrogates for presumed LTBI $(3,6-8)$. Therefore, LTBI is an operational and not a pathogenetic definition.

Since a quarter of the world's population is estimated to have LTBI, there is a large reservoir from which TB can emerge to fuel its worldwide pandemic (9). Understanding all of the immune components that result in LTBI or resistance to it, and in the continued control or possibly clearance of Mtb, is critical for insights

Conflict of interest: The authors have declared that no conflict of interest exists. Copyright: (5) 2021, American Society for Clinical Investigation.

Reference information: J Clin Invest. 2021;131(3):e136222.

https://doi.org/10.1172/JCl136222. into protective immunity to Mtb and for determining who is at risk of developing TB (10). Genetic studies indicate that Mtb may have coevolved with humans for more than 6000 years, which likely contributed to its success in intracellular survival and escape from innate and adaptive immune mechanisms (10-13). The bacterial pathogenesis, evolution, and strain diversity of Mtb have been extensively reviewed elsewhere (10-14). Based on human and nonhuman primate (NHP) studies, we here focus on new concepts and point out major knowledge gaps in efforts to understand the complexity of immune responses in LTBI.

\section{Models for human LTBI}

Animal models have provided insight into essential mechanisms of TB pathogenesis, but few reflect the heterogeneity of human responses to Mtb, particularly during the early events of control and containment in the airways (refs. 15, 16, and reviewed in refs. 17-19). NHPs, especially macaques, have been invaluable models for Mtb infection of the lung. They display the full spectrum of host responses and clinical manifestations that most closely resemble those in humans (reviewed in refs. 20, 21). Macaques differ in their susceptibility to Mtb - around $90 \%$ of rhesus and $60 \%$ of cynomolgus macaques develop TB after low-dose airway infection (20-22). Both macaque models are being used to study TB pathogenesis and TB vaccine responses, and provide important insights into $\mathrm{T}$ and $\mathrm{B}$ cell-mediated correlates and mechanisms of protection against Mtb and its progression to TB in the setting of immunosuppression (e.g., SIV infection) and T and B cell depletion (reviewed in refs. 17-21; refs. 23-26). The cynomolgus model, owing to its higher rate of Mtb control, is more suitable for investigation of the earliest events in the lung leading to granuloma development, and LTBI or progression to TB (15-18, 20-22). With sophisticated imaging, systems immunology, and computational modeling approaches (27), NHP models will continue to enhance our understanding of pathogenesis in human TB and LTBI. 
Table 1. Major human defense mechanisms in Mtb exposure and infection

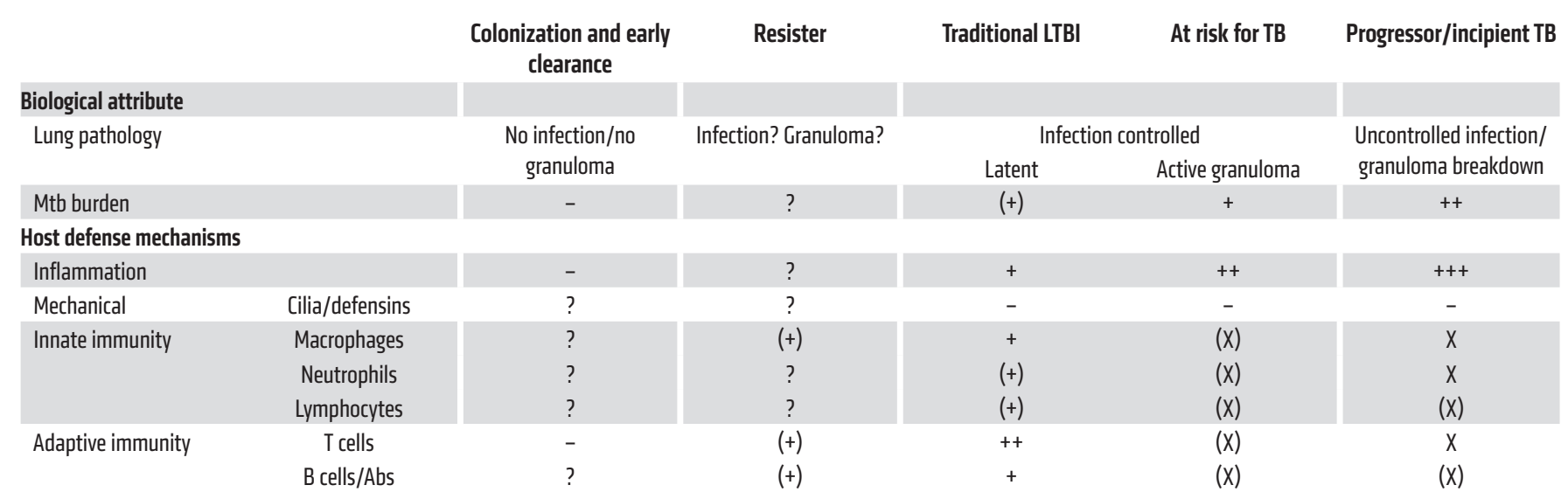

-, absent; +, present; (+), probably present but data limited; ?, unknown; $(X)$, probably failing but data limited; $X$, failure and/or imbalance.

Human granuloma models allow for analyses of early host-pathogen interactions during Mtb infection (reviewed in ref. 28). They bring together cells such as mononuclear phagocytes, lymphocytes, fibroblasts, and epithelial cells, and allow investigation of the impact of different human immune components on early granuloma formation. Mycobacterial growth inhibition assays are another tool for in vitro/ex vivo assessment of immune responses to Mtb in humans (reviewed in ref. 29). While these in vitro systems have limitations, such as short infection duration, limited cell type diversity, and inability to model kinetics of immune cell recruitment, these models likely will continue to become more sophisticated and contribute to our understanding of human granuloma formation.

\section{Development and spectrum of LTBI}

Based on animal studies, after inhalation some Mtb bacilli reach distal alveolar spaces where they are engulfed by alveolar macrophages, resident dendritic cells (DCs), and/or recruited mononuclear phagocytes (reviewed in refs. 18, 30). Infected cells travel to local lymphoid tissues (e.g., bronchus-associated lymphoid tissue or mediastinal lymph nodes) where Mtb antigens are processed and presented by DCs to initiate an adaptive immune response. In most, this results in pulmonary granuloma formation, which controls or eliminates Mtb (reviewed in refs. 4, 18). Failure of adaptive, mostly cell-mediated immune responses to control Mtb, as seen for example in newborns and advanced HIV disease, results in direct progression from infection to pulmonary or disseminated TB (reviewed in refs. 31-33).

Studies in macaques have expanded our understanding of immune mechanisms in LTBI (reviewed in refs. 17-21). These studies show that granulomas can be initiated by a single bacillus, are heterogeneous, and develop independent trajectories, with some becoming sterile, some containing small numbers of Mtb, and others progressing with necrosis and uncontrolled bacterial growth either naturally or when immune suppression is applied. NHP studies have also helped establish that controlled granulomas consist of a core of macrophages and neutrophils/ polymorphonuclear cells surrounded by $\mathrm{T}$ and $\mathrm{B}$ cells expressing a balanced panel of proinflammatory (e.g., IFN- $\gamma$, IL-17, TNF- $\alpha$ ) and antiinflammatory (e.g., IL-10, TGF- $\beta$ ) cytokines (reviewed in ref. 4), and that concurrent Mtb infection is protective against a secondary Mtb challenge (34). Understanding the differences between granulomas that control and those that do not control Mtb is a critical area of research.

In most individuals who are not overtly immune-compromised, adaptive immune responses control Mtb growth, primarily through T cells, which, through secretion of cytokines such as IFN- $\gamma$ and TNF- $\alpha$ and cytolytic function, promote the ability of macrophages to control the growth of Mtb (reviewed in refs. 18, 35). The majority (about 90\%) of these individuals do not progress from infection to disease (reviewed in refs. 3, 4). Evidence that they have been exposed to Mtb and are likely infected stems from their positive TST and/or IGRA, in which case they meet the criteria for having LTBI $(3,6-8)$. The TST is based on a delayedtype hypersensitivity response to a mixture of 100-200 denatured Mtb proteins and peptides, referred to as purified protein derivative (PPD). Because many proteins in PPD are also found in other mycobacteria, including the current $\mathrm{TB}$ vaccine strain $M$. bovis bacillus Calmette-Guérin (BCG) (36), responses to PPD may not be Mtb specific. The more Mtb-specific blood-based IGRAs measure $\mathrm{CD} 4^{+} \mathrm{T}$ cell responses to peptides from Mtb-specific proteins, such as ESAT6, CFP10, and TB10.4, which are not generated by most nontuberculous mycobacteria and BCG.

Epidemiologic and cohort studies indicate that the risk of progression from LTBI to disease is around $5 \%-10 \%$ and is greatest in the first 1-2 years after TST/IGRA conversion (37-40). This observation suggests that in recent TST/IGRA converters, progression from infection to disease reflects poor control of the initial Mtb infection, allowing continued slow Mtb replication until the uncontrolled infection becomes clinically apparent. In children, very high versus low IGRA responses can differentiate risk of progression to $\mathrm{TB}$, but the magnitude of response is of less value in adults $(41,42)$ and does not reflect mycobacterial burden or state of protective immune activation in LTBI. Some individuals progress from LTBI to TB years later, but estimates of rates vary widely (reviewed in ref. 43). Epidemiologic studies on the impact of immunosuppression (e.g., HIV infection, anti-TNF therapy, and organ or bone marrow transplantation) on people with LTBI 
estimate that only a minority develop TB (reviewed in ref. 5). Because progression is seen in non-TB-endemic settings where the risk for Mtb reinfection is low, these data suggest that those who progressed harbored viable Mtb whereas those who did not may have cleared the bacilli. Biomarker studies are making inroads into determining who is at risk for progression from LTBI to TB (reviewed in ref. 44), but prospective validation studies are needed to determine the ability of these biomarkers to estimate Mtb exposure and infection, size of mycobacterial burden, and level of protective immunity.

While some people with heavy Mtb exposure appear to resist what we define as LTBI (reviewed in ref. 45 and discussed below), many individuals with LTBI who progress to TB do not have an obvious acquired immunodeficiency or risk factor, suggesting potential undefined genetic risk factors. Higher rates of TB in monozygotic than in dizygotic twins provided evidence for a role for human genetics (46). Furthermore, Mendelian susceptibility to mycobacterial disease (MSMD) has defined the importance of the IFN- $\gamma /$ Stat1/IL-12 axis for host defenses against mycobacteria, including Mtb (47). However, genetic association studies have yet to directly link a gene, locus, or gene network with a specific mechanism to explain resistance or susceptibility to $\mathrm{TB}$ (reviewed in ref. 48). New data indicate genetic variations associated with TST conversion in Brazilian TB household contacts (49), but more studies focusing on earlier phases of TB pathogenesis, including susceptibility to Mtb infection and development of LTBI, are needed.

In TB-endemic settings the vast majority of people with LTBI are unable to pinpoint a recent $\mathrm{Mtb}$ exposure, remain well, and do not progress to TB. The term LTBI implies that small numbers of "latent" but viable Mtb bacilli are contained in granulomas and can reactivate to cause TB (reviewed in ref. 3 ). Because it is not possible to detect latent bacilli in vivo yet, we cannot parse individuals with LTBI into those harboring "latent" Mtb and those who may have cleared the bacilli. However, we know that for most the cellular immune response to Mtb that defines LTBI reflects control of exposure to and/or infection with Mtb (Table 1; and reviewed in ref. 33). We further know that LTBI comprises a spectrum of host immune responses, likely influencing the potential clearance or degree of persistent Mtb burden (reviewed in ref. 4). While not measuring Mtb directly, studies using PET-CT can provide insight into this spectrum of immune activation and its correlation with Mtb control or progression to TB. Improved understanding of all of the immune components that result in resistance, clearance, or maintenance of LTBI will enhance our insights into protective immunity to Mtb.

\section{Resistance to traditional LTBI}

In TB endemic settings or environments with heavy Mtb exposure (e.g., sharing a berthing compartment at sea with an individual with pulmonary TB), some people remain TST and/or IGRA negative (reviewed in ref. 45). Recent studies from Uganda, India, and Indonesia have extended these earlier observations of individuals who appear to resist the development of "traditional" LTBI despite extensive Mtb exposure (50-54). We estimate that $5 \%-10 \%$ of adult TB household contacts in a TB-endemic urban environment such as Kampala, Uganda remain TST/IGRA negative and clinically well after prolonged follow-up (53). Furthermore, approximately $10 \%$ of South African miners, who may have the highest Mtb exposure in the world, remain TST negative after years of working in the mines (55).

The lack of a traditional LTBI response in heavily Mtbexposed people raises several interesting immunopathogenesis questions. Do individuals who resist Mtb infection (resisters) have a unique respiratory mucosal immune response that clears Mtb from airways before it reaches the alveolus? Are innate or trained macrophages able to control Mtb without help from T cells? Do resisters have an alternative $\mathrm{T}$ cell response not measured by TST/ IGRA that clears and/or controls Mtb? Is there a role for protective $\mathrm{B}$ cell responses? Is there a role for genetics? As with traditional LTBI, the inability to detect Mtb does not allow us to determine whether and which resisters could be latently infected or may have cleared Mtb $(5,56)$. Understanding the host response and immune mechanism(s) of these LTBI resisters may identify novel protective immune responses to Mtb.

Based on cohort studies of Ugandan household contacts who were highly exposed to Mtb but remained TST and IGRA negative during an almost decade-long follow-up period, we have evidence for differences in both innate and adaptive immune responses (52, 56). Monocyte-derived macrophages from resisters and people with LTBI differed in gene expression and metabolic programs in response to Mtb, suggesting their contribution to resistance to a traditional LTBI response $(57,58)$. In addition, we found nonIFN- $\gamma$ T cell responses to the Mtb-specific proteins ESAT6 and CFP10 in resisters, while their overall $\mathrm{T}$ cell responses revealed normal IFN- $\gamma$ responses (56). These non-IFN- $\gamma$ T cell responses were associated with Mtb-specific antibody profiles and characteristics, indicating that resisters were Mtb-exposed. Among Indonesian TB household contacts, those resisting Mtb infection had evidence for trained immune responses (59). Importantly, while the cohort was small, there was no evidence that resisters were at increased risk of progression to TB, i.e., their immune responses were adequate to control their exposure to aerosolized Mtb.

Based on these data, we believe that these Ugandan resisters may have developed an alternative form of LTBI. Resisters might have enhanced macrophage capacity to control Mtb, due to either trained immunity or genetic factors, and less need for an expansive $\mathrm{T}$ cell response. Alternatively, resisters may have a unique combination of $\mathrm{B}$ and $\mathrm{T}$ cell responses that help macrophages control Mtb. Some elements of these resisters' immune responses likely are also present in subsets of people with traditional LTBI. Studies of the immune responses of well-characterized resisters from various settings may provide insights into alternative mechanisms of protection against Mtb, TB host-directed therapies, and approaches to vaccine development.

\section{T cells and LTBI}

$\mathrm{T}$ cells are critical for successful containment of Mtb by macrophages in granulomas (Figure 1), and many $\mathrm{T}$ cell subsets respond to a wide range of Mtb antigens. These subsets can broadly be defined as classical MHC-restricted T cells and donor-unrestricted T cells (DURTs), with the former responding to wide-ranging Mtb peptides (reviewed in ref. 60) and the latter to a restricted set of mostly nonprotein antigens (reviewed in ref. 61). HIV-induced $\mathrm{CD}^{+} \mathrm{T}$ cell depletion and its association with $\mathrm{TB}$ risk provide the strongest evidence for the dominant role of $\mathrm{CD} 4^{+} \mathrm{T}$ cells in 


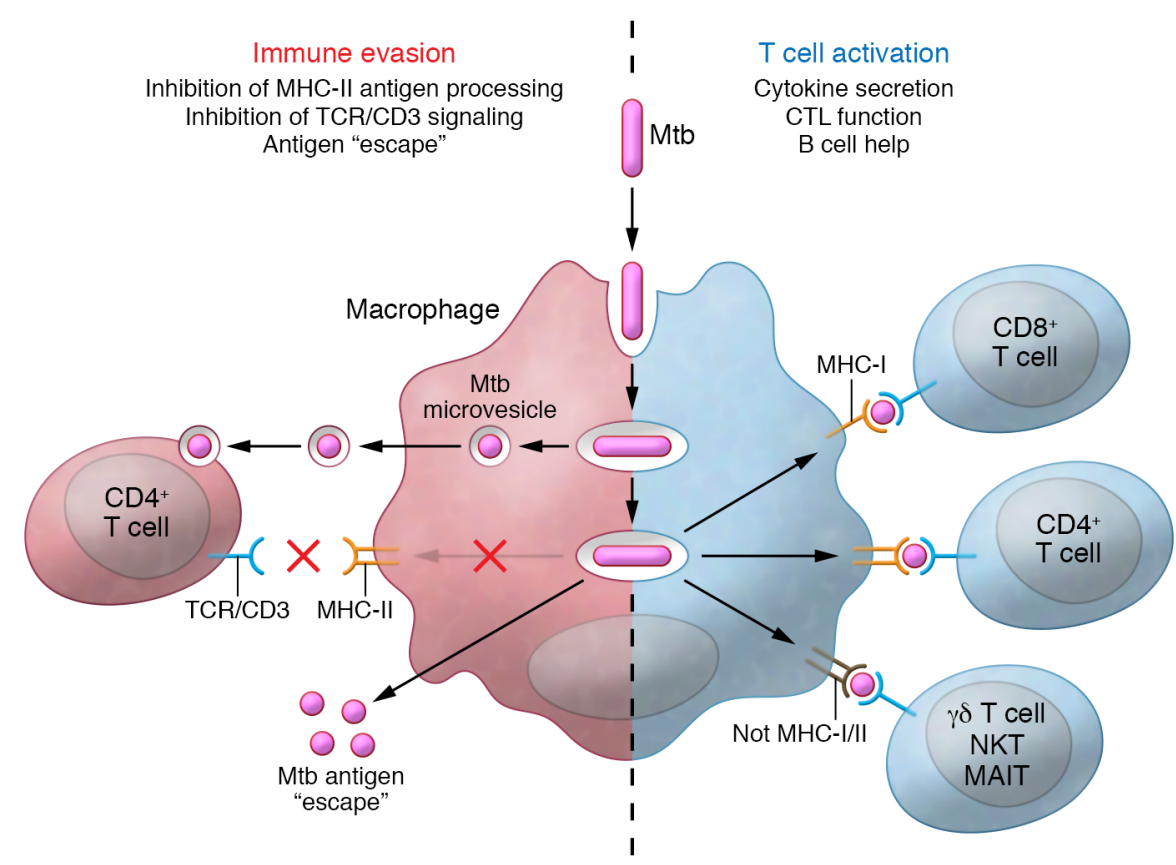

Figure 1. Evasion of $\mathrm{T}$ cell recognition versus $\mathrm{T}$ cell activation by Mtb-infected antigen-presenting cells. The paradox of the T cell response to Mtb is that, on the one hand, Mtb antigens, when appropriately processed by an activated antigen-presenting cell, elicit a broad T cell response in a person with LTBI. This involves many $T$ cell subsets responding to a wide range of antigens. These Mtb-activated T cells secrete predominantly Th1 cytokines and chemokines, possess cytotoxic $T$ lymphocyte (CTL) function, and can provide help to B cells. On the other hand, Mtb harbored by macrophages can use a variety of mechanisms to interfere with $T$ cell recognition. These mechanisms have primarily been identified for $\mathrm{CD}^{+} \mathrm{T}$ cells and include inhibition of MHC-II antigen processing, antigen escape, and inhibition of T cell receptor-CD3 signaling by Mtb glycolipids, but some may also apply to other T cell subsets. MAIT, mucosal-associated invariant T cell.
MHC-I-restricted CD8 ${ }^{+} \mathrm{T}$ cells responsive to Mtb are found in peripheral blood and in the bronchoalveolar lavage fluid of humans and NHPs with LTBI (ref. 69 and reviewed in refs. $19,35,70)$. Recent computational modeling studies based on LTBI in NHP data suggest that multifunctional $\mathrm{CD}^{+} \mathrm{T}$ cells have a central role in preventing Mtb dissemination (27). Furthermore, DURTs that respond to Mtb may have a role in innate responses to $\mathrm{Mtb}$ (reviewed in refs. 35, 61). These include $\gamma-\delta$ T cells, CD1d-restricted natural killer (NK) $\mathrm{T}$ cells, and mucosal-associated invariant T cells. However, while DURTs are biologically intriguing, their specific roles in protecting against Mtb still need to be characterized. Despite our broad knowledge of cell-mediated immunity in LTBI, many knowledge gaps remain: (a) What $\mathrm{T}$ cell protective antigens are most relevant to the recognition of infected cells? (b) Does Mtb's ability to inhibit antigen processing limit antigens presented by infected cells? (c) How do BCG vaccination and exposure to environmental mycobacteria modulate $\mathrm{T}$ cell responses after Mtb infection? (d) How do $\mathrm{CD}^{+} \mathrm{T}$ cells contribute to protective immunity in LTBI? (e) How do the granuloma milieu and architecture impact T cell function? controlling Mtb (reviewed in ref. 33). Murine MHC-II knockout and NHP CD4 $4^{+} \mathrm{T}$ cell depletion studies further support this central role of MHC-II-restricted CD4 ${ }^{+} \mathrm{T}$ cells (reviewed in ref. 35). Polyfunctional $\mathrm{CD}^{+}{ }^{+} \mathrm{T}$ cells expressing IFN- $\gamma$, TNF- $\alpha$, and IL- 2 are associated with protective responses (62), and effector/memory $\mathrm{CD} 4^{+} \mathrm{T}$ cells responsive to $\mathrm{Mtb}$ antigens are found in the bronchoalveolar lavage fluid of people with LTBI (63). In addition, CD $4^{+}$ Treg and Th17 responses to Mtb are found in LTBI, but their role in controlling Mtb infection is less clear.

Mtb-activated human CD4 ${ }^{+} \mathrm{T}$ cells help macrophages control intracellular mycobacteria through secretion of cytokines and cytotoxic T lymphocyte function (ref. 64 and reviewed in refs. 35, 65). In addition to these direct effector roles, $\mathrm{CD} 4^{+} \mathrm{T}$ cell subsets also provide important helper functions for other immune cells involved in LTBI, including help for $\mathrm{CD}^{+} \mathrm{T}$ cell and DURT expansion, and for antibody production by B cells (reviewed in refs. 66, 67). While the central role of $\mathrm{CD} 4^{+} \mathrm{T}$ cells in LTBI and protection against TB is well established, the key Mtb antigens recognized by protective $\mathrm{T}$ cells have not been identified. CD $4^{+} \mathrm{T}$ cells (and CD8 ${ }^{+}$ T cells; see below) from people with LTBI demonstrate broad reactivity to Mtb peptides $(60,68)$, but only a limited number of antigens are recognized by most individuals with LTBI. Antigens expressed by MHC molecules on Mtb-infected cells remain largely unknown. Identifying these antigens is essential to define the key protective T cells for LTBI.

\section{Mtb's evasion of T cell recognition}

Elegant cell biology and functional studies have defined a number of molecular mechanisms used by Mtb to resist innate immune mechanisms in macrophages and DCs, including disruption of progression to phagolysosome fusion, and resisting of killing by superoxide, autophagy, and apoptosis (reviewed in refs. 30, 35, 65, $71,72)$. Mtb can also indirectly and directly interfere with recognition of infected cells by $\mathrm{CD} 4^{+} \mathrm{T}$ cells (Figure 1). For example, Mtb lipoproteins can activate TLR2 signaling in macrophages, which inhibits IFN- $\gamma$-driven expression of MHC-II molecules (73); Mtb's secreted protein EsxH can interfere with $\mathrm{CD}^{+} \mathrm{T}$ cell activation (74); and Mtb-infected DCs can export antigens to uninfected cells, thereby limiting their antigen presentation to and activation of $\mathrm{CD} 4^{+} \mathrm{T}$ cells $(75)$.

Mtb resides in macrophage phagosomes, which resemble an endosomal recycling compartment that traffics molecules and bacterial vesicles. Release of bacterial microvesicles allows Mtb products, which include lipids, proteins, and glycolipids such as lipoarabinomannan (LAM), to reach $\mathrm{T}$ cells in the proximity of infected cells (76-78). Exposure of CD4 ${ }^{+} \mathrm{T}$ cells to LAM or LAM-containing microvesicles inhibits proximal $\mathrm{T}$ cell receptor-CD3 signaling, which induces GRAIL (gene regulating anergy in lymphocytes), rendering LAM-exposed $\mathrm{CD} 4^{+} \mathrm{T}$ cells anergic (79). Similar inhibitory mechanisms are likely applicable to $\mathrm{CD} 8^{+} \mathrm{T}$ cells, and DURTs, since they all rely on CD3 for activation. Despite these known 
direct and indirect mechanisms of Mtb interference with T cell recognition of infected cells, questions remain: (a) Do these evasion mechanisms impact non-CD4 ${ }^{+} \mathrm{T}$ cells? (b) During which stages of Mtb infection and disease do they affect the immune response? (c) Which of these different $\mathrm{T}$ cell evasion mechanisms dominates, and at what stage of Mtb pathogenesis in vivo?

\section{Antibodies and B cells in LTBI}

Antibodies may contribute to long-term Mtb control in LTBI (reviewed in refs. 80-84). Serum IgG from individuals exposed to or latently infected with Mtb can be protective in vitro and in vivo against Mtb (85-87). Mtb resisters carry IgM against ESAT6 and CFP10 and other Mtb antigens and have class-switched IgG antibody responses, suggesting a role in these persistently TST/ IGRA-negative but heavily Mtb-exposed individuals (56). In contrast, few studies support a protective role for anti-Mtb antibodies from TB patients (88).

Antibodies can bind mycobacterial surface molecules and interact with Fc receptors ( $\mathrm{FcRs}$ ) on phagocytes (reviewed in refs. 80-84). While binding to surface molecules can activate complement and prevent bacterial adhesion and invasion of host cells, subclasses or isotypes and their distinct Fc glycosylation profiles can influence FcR-mediated effects, including inflammatory versus noninflammatory responses. Through Fc $\gamma \mathrm{R}$, mycobacterial multi- and single-antigen-specific polyclonal IgG from asymptomatic Mtb-exposed and infected people can enhance Mtb phagocytosis and growth inhibition, and antibody-dependent cellular cytotoxicity $(85,87,89)$. Enhanced cytotoxic responses mediated mostly by FcyRIIIa (CD16) and NK cells were also observed in LTBI $(87,90)$. These data demonstrate the important interplay between antibodies and the innate immune system in LTBI.

The range of mycobacterial antigens targeted by protective antibodies remains poorly understood. Transfer studies with murine IgG or IgA monoclonal antibodies (mAbs) in Mtb-infected mice suggest that antibodies targeting the surface glycan arabinomannan (AM), the glycolipid lipoarabinomannan (LAM), the surface protein heparin-binding hemagglutinin (HBHA), the heat shock protein HspX, and the 38-kDa adhesion protein PstS1 might be protective (reviewed in ref. 91). Vaccination with AM and antigen 85 followed by passive transfer of antibodies was moderately protective against Mtb in mice (92). In humans, antibodies against antigen 85 and AM/LAM appear to be protective $(85,93,94)$, but experimental data with human $\mathrm{mAbs}$ remain scarce.

Attempts to identify significantly different antigen-specific antibody responses in LTBI versus TB are ongoing, but have provided few conclusions to date $(95,96)$. In both NHPs and humans, antibody responses to Mtb are heterogeneous (85, 97-101), likely because of granuloma heterogeneity (reviewed in ref. 19), large numbers of differentially expressed Mtb antigens (102), and/or prior exposure to BCG and/or nontuberculous mycobacteria (85, $100,103)$. This heterogeneity contributes to the challenges of delineating specific protective antibodies against Mtb.

A limited number of functional human multi- or singleantigen-specific polyclonal antibody studies have been performed $(56,85-88,104)$. Protective ex vivo efficacy was reversed when total serum IgG from asymptomatic health care workers was preabsorbed with Mtb (86). We found that anti-AM IgG iso- lated from high-titer asymptomatic TST-positive individuals was protective in vitro and in vivo (85). In line with serum anti-AM IgG studies from an adult BCG vaccination trial (100), our data further suggested the importance of targeting specific glycan epitopes within AM and support the protective role of IgG to certain Mtb surface antigens and epitopes.

Efforts to generate human mAbs against Mtb-specific antigens/epitopes are ongoing $(105,106)$, which will help define the roles of variable and $\mathrm{Fc}$ domains. Human $\mathrm{mAb}$ isotypes against LAM and HBHA generated from plasmablasts and memory B cells of TB patients and Mtb-exposed health care workers demonstrated different effector functions (105); IgG enhanced and IgA inhibited Mtb uptake by human lung epithelial cells and macrophages, irrespective of the target, although neither differences in FcR expressions between these cell types nor effects on intracellular Mtb growth were taken into consideration.

Antibodies in the airways could serve as a first line of defense against inhaled Mtb (Figure 2). For example, secretory IgA could bind to Mtb antigens and thereby prevent Mtb adhesion to and infection of airway cells, while in parallel facilitating elimination of Mtb via mucociliary clearance. Passive transfer studies support a protective role of poly- and monoclonal IgG and IgA against Mtb in the airways (reviewed in refs. 83, 91; refs. 85, 86). Polyfunctional Th17 cells, IL-10, and increased airway IgA levels were associated with protection against Mtb in NHPs mucosally vaccinated with BCG (107), and mucosal vaccination of mice and NHPs with the MTBVAC vaccine indicated a role of mucosal secretory antibodies against Mtb (108). The role of antibodies after intravenous BCG, shown to be more protective than airway vaccination, remains to be determined (109).

Antibodies also can synergize with $\mathrm{T}$ cells in controlling Mtb (92), and, in addition to being influenced by T cells (reviewed in ref. 66), B cells may regulate $\mathrm{T}$ cell and cytokine responses during Mtb infection, thereby influencing inflammation and granuloma formation (reviewed in refs. 110, 111). B cells are present in the granulomatous lesions of Mtb-infected mice, non-human primates, and humans. Although inconsistent results of murine studies have led to controversy regarding the protective effects of B cells in Mtb infection (reviewed in ref. 110), recent data show an association of smaller lung B cell follicles with increased Mtb susceptibility in male versus female mice (112), and NHP studies support the beneficial effects of B cells in the lung. Despite a lack of difference in outcome between B cell-depleted and nondepleted Mtb-infected cynomolgus macaques, B cell depletion influenced local $\mathrm{T}$ cell and cytokine responses, resulting in increased Mtb burden at the granuloma level (113). Expanded B cell follicles in the lungs of Mtb- and SIV-coinfected rhesus macaques were also associated with lack of progression to TB (24).

In humans, household contacts with LTBI and TB patients were shown to have atypical B cell phenotypes associated with a compromised $\mathrm{T}$ cell response, which, in TB patients, resolved after antituberculous treatment (114). These atypical B cells showed diminished proliferation and immunoglobulin and cytokine production, supporting their lack of function in TB. Circulating naive B cells are reduced in LTBI, possibly as a result of sequestration at the site of infection (90). B cells form prominent aggregates in the lungs of Mtb-infected humans, NHPs, and mice $(24,115-118)$. Nevertheless, 
A

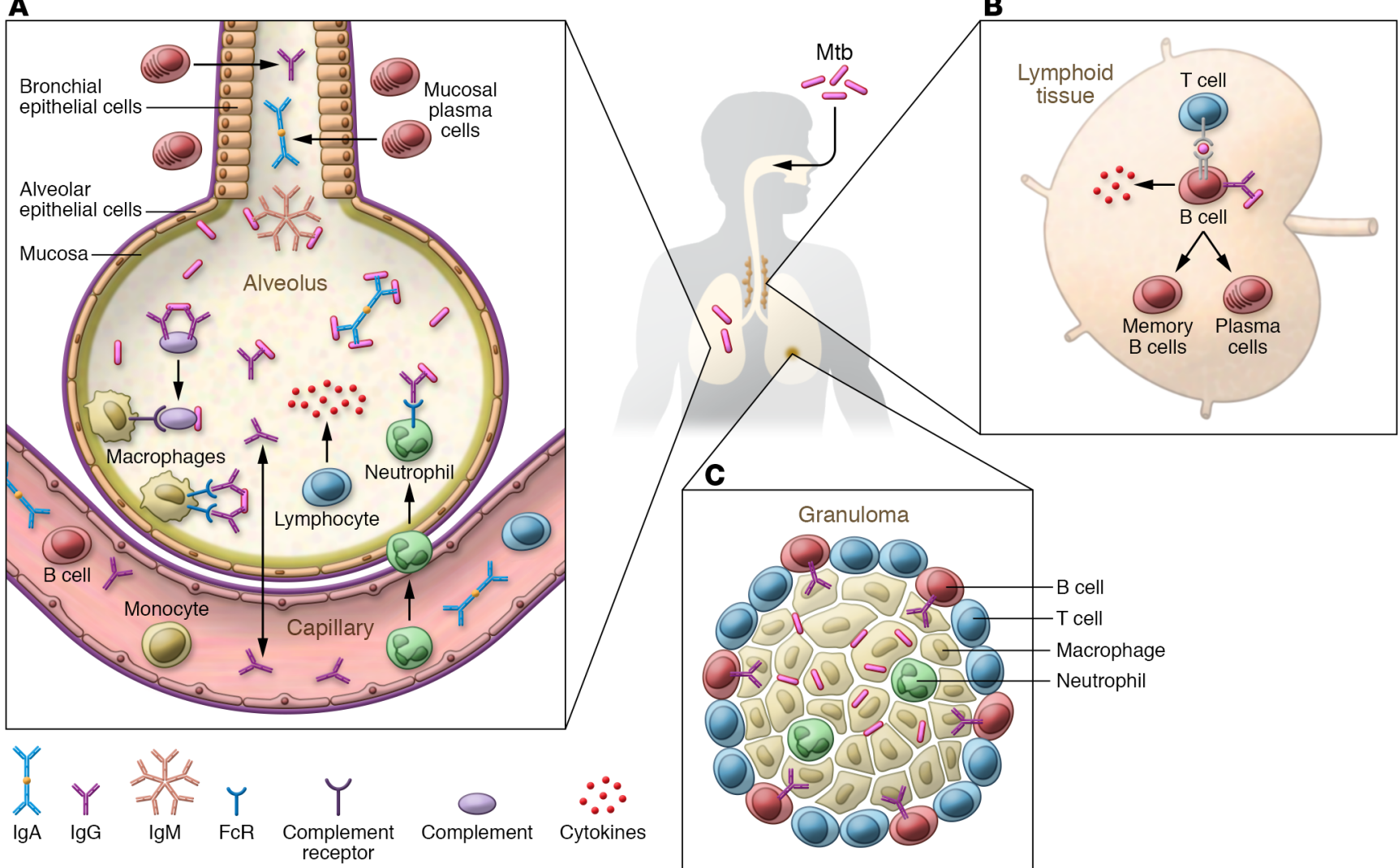

Figure 2. Potential protective roles of antibodies and B cells in the lung during both initial Mtb exposure and LTBI. (A) Antibody isotypes (IgA, IgG, and IgM) could impact Mtb in the lower airways through binding, opsonization, complement activation, and FcR-mediated enhanced phagocytosis and intracellular growth reduction by phagocytes. (B) B cells located in germinal centers of lymphoid tissues could control infection through (i) enhancing antigen presentation to T cells; (ii) production of helper cytokines for T cells; and (iii) generation of antibodies that could modulate innate and adaptive immune responses. (C) Both the presence of B cells and the pro- and antiinflammatory capacities of antibodies could influence the formation of functional granulomas and thereby contribute to the control of Mtb.

because of the conflicting associations with disease outcome, the role of these lung B cell aggregates remains to be determined.

Overall, many questions remain regarding the roles of antibodies and B cells in the defense against Mtb: (a) What are the critical antigens in antibody-mediated immunity against Mtb? (b) How do epitope specificity and Fc glycosylation influence success and failure of Mtb control? (c) What are the protective roles and mechanisms of IgG, IgA, and IgM during Mtb exposure and infection? (d) Do antibodies have direct effects on Mtb? (e) What are the essential interactions between the humoral and other immune arms in the defense against Mtb? (f) What role do B cells and pulmonary B cell aggregates have in Mtb infection? A better understanding of these roles will inform immunotherapy and TB vaccine development.

\section{Innate immune responses and LTBI}

Innate immune cells, both lymphoid and myeloid, have a central role in the host response to Mtb (reviewed in refs. 30, 35, 61, 119). Recent studies have expanded our understanding of the range of innate cells, such as the myeloid-derived polymorphonuclear cells (PMNs) and innate lymphoid cells and DURTs (discussed above), involved in responses to Mtb and influencing the complexity of macrophage responses. Nevertheless, whereas the centrality of macro- phages as nidus and site of Mtb control in LTBI is well established, the role of other innate cells in LTBI is less clear. In vivo innate responses upon Mtb infection can only be studied in experimental animals, and in vitro studies of macrophage functions are primarily performed with bone marrow-derived macrophages from C57BL/6 mice, considered resistant to Mtb infection, and with human blood monocyte-derived macrophages and macrophage cell lines. Where results from these studies fit in the spectrum from Mtb exposure to LTBI and TB in humans is not straightforward.

As a facultative intracellular pathogen in macrophages, Mtb depends on phagocytosis for host cell entry. Thus, the receptor repertoire of these cells defines infectivity and shapes downstream host responses. Upon inhalation, Mtb trapped in the alveolar surfactant phospholipid layer can be bound by surfactant proteins $\mathrm{A}$ and $\mathrm{D}$ for indirect phagocytosis by alveolar macrophages, a defense mechanism deficient in the elderly (120). Once phagocytosed, Mtb proliferates in macrophages by interfering with phagosome maturation through cell wall glycolipids $(72,121,122)$. The mycobacterial phagosome communicates dynamically with endosomes and delivers mycobacterial antigens into the lysosomal degradation pathway for antigen processing. During phagocytosis, Mtb also triggers a set of pattern recognition receptors, which induce both proinflammatory (IL-1, 


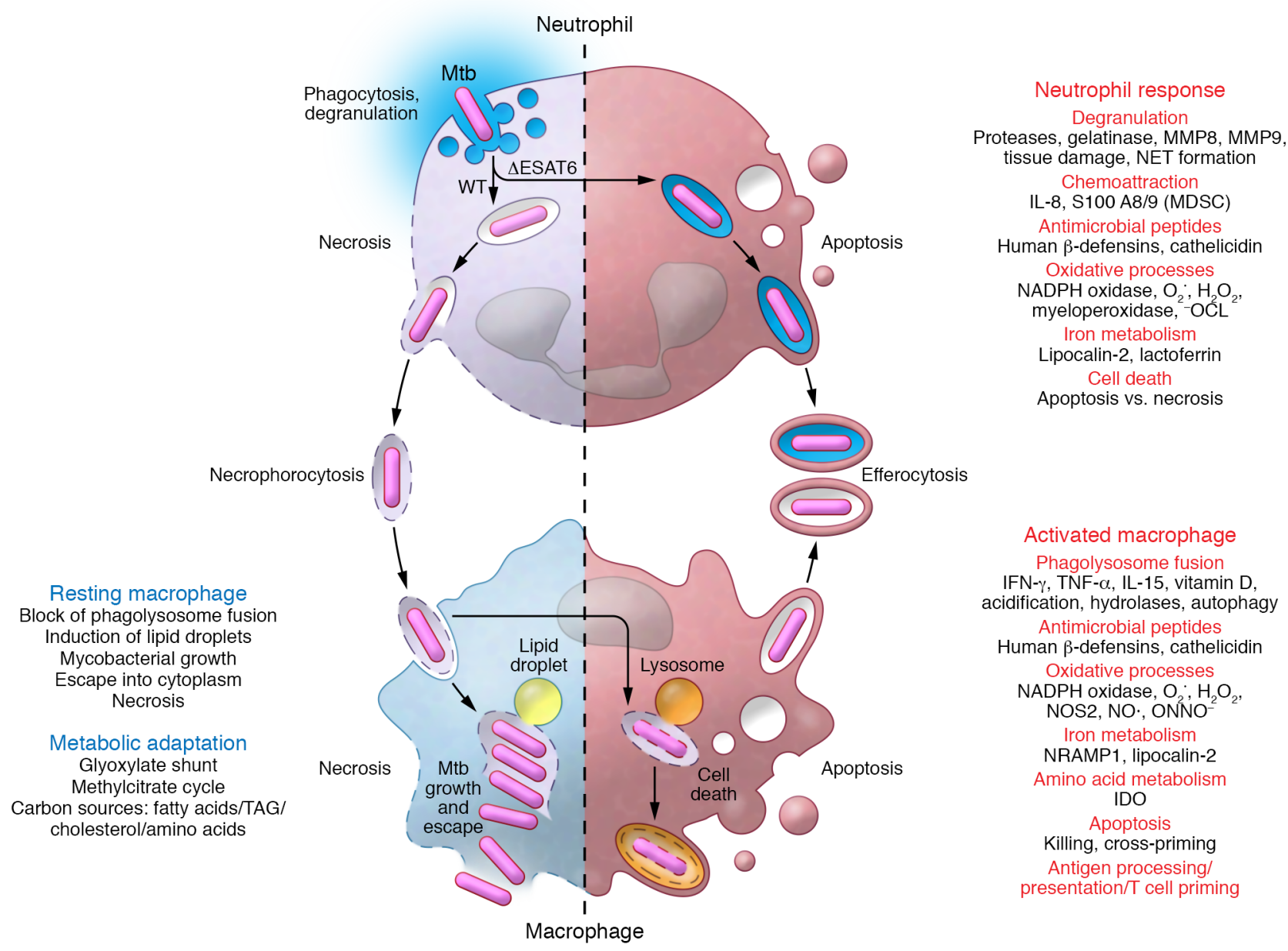

Figure 3. Interactions between infected PMNs and macrophages can determine the balance of exacerbating versus protective host responses in LTBI. Wild-type but not attenuated Mtb drives PMNs into necrotic cell death in a myeloperoxidase-dependent manner. Uptake of Mtb together with necrotic PMNs by resting macrophages further promotes mycobacterial propagation and macrophage necrosis, releasing Mtb for another round of intracellular replication and macrophage death. In contrast, immune activation in LTBI equips macrophages with a potent antimicrobial armamentarium to control and possibly eliminate Mtb. MDSC, myeloid-derived suppressor cells; NET, neutrophil extracellular traps; -OCL, hypochlorite.

IL-12/-23, TNF- $\alpha$, and type I IFNs) and antiinflammatory (IL-10) responses (reviewed in refs. 123-125). Mtb cell wall glycolipids interacting with C-type lectins can switch a proinflammatory to an antiinflammatory IL-10 response $(122,126)$. Alveolar macrophages exhibit a predominantly antiinflammatory M2 phenotype, which Mtb can use to establish its intracellular niche (reviewed in refs. 72, 127).

Alveolar macrophages also transport Mtb into the bronchusassociated lymphoid tissue, where, in LTBI, they transfer antigens to DCs to trigger adaptive $\mathrm{T}$ cell responses that help control Mtb growth (reviewed in ref. 128). Recent studies suggest that group 3 innate lymphoid cells (ILC3s) are involved in Mtb control (129). These cells were associated with enhanced alveolar macrophage recruitment in the lungs of Mtb-infected mice and, when depleted, reduced bacterial control. In TB patients, ILC3 accumulated in the lungs and were depleted in the blood with normalization after TB treatment (129), but their role in LTBI remains to be determined. High levels of circulating NK cells in LTBI may also play a role in controlling Mtb during LTBI, which is further supported by the observation that NK cell levels are low in TB and return to baseline after TB treatment (90).
In LTBI, immune activation by IFN- $\gamma$, TNF- $\alpha$, and autocrine IL-15 (probably reinforced by vitamin $\mathrm{D}_{3}$ ) can enhance Mtb control by accelerating phagosome maturation, production of microbicidal effectors, augmented glycolysis, and induced autophagy (130-132). The relevance of autophagy as an antiMtb effector of activated macrophage remains to be determined (133).

Prior pathogen exposure can train innate immunity. For example, BCG vaccination can epigenetically prime NK cells and monocytes/macrophages for a more focused secondary response (reviewed in ref. 134). Distinct innate immune cell and cytokine responses in Indonesian TB household contacts support a role for trained immunity in early clearance of Mtb in humans (59). In mice infected intravenously with BCG or Mtb, IFN- $\gamma$ was found to be an important factor in regulating macrophage trained immunity by enhancing myelopoiesis and expansion of lineage ${ }^{-} \mathrm{CKit}^{+} \mathrm{Sca}^{+}$(LKS) bone marrow stem cells $(135,136)$. Mycobacterial interaction with LKS leads to innate imprinting of myeloid cells by altering their epigenetic profile, thereby rendering mature macrophages more effective against Mtb and likely contributing to trained immunity in LTBI (137). 
The role(s) of PMNs in Mtb pathogenesis is an active area of research. In Mtb-resistant mice, numbers of infected PMNs are only transiently increased following infection (30). In contrast, susceptible mouse strains such as $\mathrm{C} 3 \mathrm{HeB} / \mathrm{FeJ}$ mice and NOS2- or Atg5-knockout mice had PMN infiltrates associated with exacerbation of necrotic granulomas and earlier death due to higher Mtb loads $(133,138,139)$. These latter data indicate that NOS2 and Atg5 are essential to restrict Mtb growth, likely through interference with PMN influx and associated pathology. Recent data from Mtb-infected mice further suggest that long-lived PMNs can accumulate in the lungs and serve as an intracellular niche for Mtb growth and persistence (140).

Necrotic PMN-laden granulomas in susceptible mice share features with those found in infected NHPs and in TB patients, where PMNs represent the dominant Mtb-infected cell population $(141,142)$. The pro- and antiinflammatory cytokine profiles of PMNs in Mtb-infected NHP granulomas suggest that the cells have an important immunoregulatory role. The abundance of PMNs in human and NHP TB lesions, together with a PMN-associated transcriptomic signature in PBMCs of TB patients (143), and enhanced PMN-driven inflammation in TB patients with type 1 diabetes $(144,145)$, links PMNs with disease, rather than LTBI. However, it is not known whether PMNs drive disease progression, or whether they are attracted to granulomas as a result of failed Mtb control. In NHPs, PMNs are part of stable Mtb granulomas, and uptake of infected PMNs by DCs facilitates T cell priming in mice (146), suggesting a protective role. It therefore remains unclear whether PMNs, with the right balance of inflammatory effects, contribute to Mtb control after initial exposure and in LTBI.

In vitro, virulent Mtb strains drive PMNs quickly into necrotic cell death $(147,148)$. Necrotic Mtb-infected PMNs release neutrophil extracellular traps as an antimicrobial effector but do not kill Mtb. Instead, clearance of necrotic Mtb-infected PMNs by macrophages promotes mycobacterial growth in these more sustainable host cells. Subsequently, infected macrophages also succumb to necrotic cell death and release mycobacteria to infect new phagocytes, thereby continuing the infectious cycle. IL-8 from infected PMNs and macrophages feeds an influx of PMNs and sustains a cycle of host cell necrosis, necrophorocytosis (phagocytic removal of necrotic cellular debris), and bacterial growth in TB lesions (reviewed in ref. 149).

Mtb-triggered PMN necrosis requires myeloperoxidasederived (MPO-derived) reactive oxygen species. Inhibition of MPO rescues infected PMNs from necrosis and restores the macrophage's ability to control Mtb upon efferocytosis of infected but apoptotic PMNs (148). Therefore, MPO and other factors associated with $\mathrm{PMN}$-driven pathology may represent intriguing targets for host-directed therapy for TB, shifting the balance back toward LTBI (reviewed in refs. 35, 149-152). Yet only interactions between infected resting macrophages and PMNs have been studied (Figure 3). Thus, the impact of macrophage activation for dealing with infected PMNs remains to be determined (72). Overall, many questions on the role of innate cells in LTBI remain, including the role of trained immunity, macrophage heterogeneity and activation in granulomas, Mtb's metabolic state, and the protective versus detrimental role of PMNs.

\section{Conclusions}

In most Mtb-infected individuals, LTBI is established through finely regulated immune responses. Summarizing known facts and important areas of LTBI research in humans and NHPs, we have pointed out critical gaps in understanding how the immune system protects against or controls Mtb. While the interaction between activated macrophages and $\mathrm{CD}^{+} \mathrm{T}$ cells is central for Mtb control in LTBI, recent discoveries reveal a more complex picture with roles for genetic factors, other $\mathrm{T}$ cell subsets, innate lymphoid cells, B cells and antibodies, trained immunity, and possibly more. Some host defenses may promote excessive inflammation, and, if not regulated properly, exacerbate pathology and facilitate progression to disease and Mtb transmission. LTBI and variants thereof, as seen in resisters, rely on both innate and adaptive immunity. The goal of parsing LTBI is to identify the immune mechanisms of the more than $90 \%$ who successfully control Mtb versus the few at risk for disease. Given our inability to distinguish who harbors dead versus live bacilli, and determine Mtb burden, LTBI remains an operational definition, hampering the triaging of care to those at greatest risk for progression to TB. Given the difficulty in identifying and preventing acute exposure and infection with Mtb in humans, animal and careful observational human studies are needed to determine the essential local immune responses necessary for elimination or long-term control of this wily pathogen with its plethora of immune evasion mechanisms.

\section{Acknowledgments}

This work was supported in part by funds from the NIH/National Institute of Allergy and Infectious Diseases to JMA (AI146329, AI127173, and AI117927) and to WHB (AI125642, AI124348, AI124348, AI147319, and contract 75N93019C00071), and by grants from the Leibniz Research Alliance INFECTIONS'21, Leibniz Science Campus Evolung, the German Science Foundation (IRTG 1911; Scha 514 5-1), and the Ministry of Education and Research (German Center for Infection Research, TB-Sequel) to UES.

Address correspondence to: Jacqueline M. Achkar, Departments of Medicine and of Microbiology and Immunology, Albert Einstein College of Medicine, 1300 Morris Park Avenue, Block Building, Room 115, Bronx, New York 10461, USA. Phone: 718.430.8763; Email: jacqueline.achkar@einsteinmed.org.
1. WHO. Global Tuberculosis Report 2019. World Health Organization; 2019.

2. Huang L, et al. Mycobacterium tuberculosis: bacterial fitness within the host macrophage. Microbiol Spectr. 2019;7(2):10.1128/microbiolspec. BAI-0001-2019.

3. Getahun H, et al. Latent Mycobacteri- um tuberculosis infection. $N$ Engl J Med. 2015;372(22):2127-2135.

4. Lin PL, Flynn JL. The end of the binary era: revisiting the spectrum of tuberculosis. JImmunol. 2018;201(9):2541-2548.

5. Behr MA, et al. Is Mycobacterium tuberculosis infection life long? BMJ. 2019;367:15770.
6. WHO. Latent Tuberculosis Infection: Updated and Consolidated Guidelines for Programmatic Management. Geneva: World Health Organization; 2018. License: CC BY-NC-SA 3.0 IGO.

7. Lewinsohn DM, et al. Official American Thoracic Society/Infectious Diseases Society of America/ Centers for Disease Control and Prevention 
Clinical Practice Guidelines: Diagnosis of Tuberculosis in Adults and Children. Clin Infect Dis. 2017;64(2):111-115.

8. Mack U, et al. LTBI: latent tuberculosis infection or lasting immune responses to M. tuberculosis? A TBNET consensus statement. Eur Respir J. 2009;33(5):956-973.

9. Houben RM, Dodd PJ. The global burden of latent tuberculosis infection: a re-estimation using mathematical modelling. PLoS Med. 2016;13(10):e1002152.

10. Gagneux S. Ecology and evolution of Mycobacterium tuberculosis. Nat Rev Microbiol. 2018;16(4):202-213.

11. Orgeur M, Brosch R. Evolution of virulence in the Mycobacterium tuberculosis complex. Curr Opin Microbiol. 2018;41:68-75.

12. Menardo F, et al. The molecular clock of Mycobacterium tuberculosis. PLoS Pathog. 2019;15(9):e1008067.

13. Saelens JW, et al. Mycobacterial evolution intersects with host tolerance. Front Immunol. 2019;10:528.

14. Ehrt S, et al. Metabolic principles of persistence and pathogenicity in Mycobacterium tuberculosis. Nat Rev Microbiol. 2018;16(8):496-507.

15. Lin PL, et al. Quantitative comparison of active and latent tuberculosis in the cynomolgus macaque model. Infect Immun. 2009;77(10):4631-4642.

16. Coleman MT, et al. Early changes by (18)Fluorodeoxyglucose positron emission tomography coregistered with computed tomography predict outcome after Mycobacterium tuberculosis infection in cynomolgus macaques. Infect Immun. 2014;82(6):2400-2404.

17. Bucsan AN, et al. The current state of animal models and genomic approaches towards identifying and validating molecular determinants of Mycobacterium tuberculosis infection and tuberculosis disease. Pathog Dis. 2019;77(4):ftz037.

18. Sia JK, Rengarajan J. Immunology of Mycobacterium tuberculosis infections. Microbiol Spectr. 2019;7(4):10.1128/microbiolspec.GPP3-0022-2018.

19. Cadena AM, et al. Heterogeneity in tuberculosis. Nat Rev Immunol. 2017;17(11):691-702.

20. Pena JC, Ho WZ. Monkey models of tuberculosis: lessons learned. Infect Immun. 2015;83(3):852-862.

21. Scanga CA, Flynn JL. Modeling tuberculosis in nonhuman primates. Cold Spring Harb Perspect Med. 2014;4(12):a018564.

22. Maiello P, et al. Rhesus macaques are more susceptible to progressive tuberculosis than cynomolgus macaques: a quantitative comparison. Infect Immun. 2018;86(2):e00505-17.

23. Bucsan AN, et al. Mechanisms of reactivation of latent tuberculosis infection due to SIV coinfection. J Clin Invest. 2019;129(12):5254-5260.

24. Foreman TW, et al. CD4+ T-cell-independent mechanisms suppress reactivation of latent tuberculosis in a macaque model of HIV coinfection. Proc Natl Acad Sci U S A. 2016;113(38):E5636-E5644.

25. Diedrich CR, et al. SIV and Mycobacterium tuberculosis synergy within the granuloma accelerates the reactivation pattern of latent tuberculosis. PLoS Pathog. 2020;16(7):e1008413.
26. Lin PL, Flynn JL. CD8 T cells and Mycobacterium tuberculosis infection. Semin Immunopathol. 2015;37(3):239-249.

27. Wessler T, et al. A computational model tracks whole-lung Mycobacterium tuberculosis infection and predicts factors that inhibit dissemination. PLoS Comput Biol. 2020;16(5):e1007280.

28. Elkington $P$, et al. In vitro granuloma models of tuberculosis: potential and challenges. JInfect Dis. 2019;219(12):1858-1866.

29. Tanner R, et al. In vitro mycobacterial growth inhibition assays: a tool for the assessment of protective immunity and evaluation of tuberculosis vaccine efficacy. Vaccine. 2016;34(39):4656-4665.

30. Srivastava S, et al. Beyond macrophages: the diversity of mononuclear cells in tuberculosis. Immunol Rev. 2014;262(1):179-192.

31. Tornheim JA, Dooley KE. Tuberculosis associated with HIV infection. Microbiol Spectr. 2017;5(1):TNMI7-0028-2016.

32. Boisson-Dupuis $S$, et al. Inherited and acquired immunodeficiencies underlying tuberculosis in childhood. Immunol Rev. 2015;264(1):103-120.

33. Jasenosky LD, et al. T cells and adaptive immunity to Mycobacterium tuberculosis in humans. Immunol Rev. 2015;264(1):74-87.

34. Cadena AM, et al. Concurrent infection with Mycobacterium tuberculosis confers robust protection against secondary infection in macaques. PLoS Pathog. 2018;14(10):e1007305.

35. Mayer-Barber KD, Barber DL. Innate and adaptive cellular immune responses to Mycobacterium tuberculosis infection. Cold Spring Harb Perspect Med. 2015;5(12):a018424.

36. Cho YS, et al. Deciphering the proteome of the in vivo diagnostic reagent "purified protein derivative" from Mycobacterium tuberculosis. Proteomics. 2012;12(7):979-991.

37. Reichler MR, et al. Risk factors for tuberculosis and effect of preventive therapy among close contacts of persons with infectious tuberculosis. Clin Infect Dis. 2020;70(8):1562-1572.

38. Sloot R, et al. Risk of tuberculosis after recent exposure. A 10-year follow-up study of contacts in Amsterdam. Am J Respir Crit Care Med. 2014;190(9):1044-1052.

39. Reichler MR, et al. Risk and timing of tuberculosis among close contacts of persons with infectious tuberculosis. J Infect Dis. 2018;218(6):1000-1008.

40. Fox GJ, et al. Household-contact investigation for detection of tuberculosis in Vietnam. N Engl J Med. 2018;378(3):221-229.

41. Andrews JR, et al. Serial QuantiFERON testing and tuberculosis disease risk among young children: an observational cohort study. Lancet Respir Med. 2017;5(4):282-290.

42. Gupta RK, et al. Quantitative IFN- $\gamma$ release assay and tuberculin skin test results to predict incident tuberculosis. A prospective cohort study. Am J Respir Crit Care Med. 2020;201(8):984-991.

43. Menzies NA, et al. Progression from latent infection to active disease in dynamic tuberculosis transmission models: a systematic review of the validity of modelling assumptions. Lancet Infect Dis. 2018;18(8):e228-e238.

44. Ahmed M, et al. Immune correlates of tuberculosis disease and risk translate across species.
Sci Transl Med. 2020;12(528):eaay0233.

45. Simmons JD, et al. Immunological mechanisms of human resistance to persistent Mycobacterium tuberculosis infection. Nat Rev Immunol. 2018;18(9):575-589.

46. Comstock GW. Tuberculosis in twins: a reanalysis of the Prophit survey. Am Rev Respir Dis. 1978;117(4):621-624.

47. Bustamante J, et al. Mendelian susceptibility to mycobacterial disease: genetic, immunological, and clinical features of inborn errors of IFN- $\gamma$ immunity. Semin Immunol. 2014;26(6):454-470.

48. McHenry ML, et al. Genetics and evolution of tuberculosis pathogenesis: New perspectives and approaches. Infect Genet Evol. 2020;81:104204.

49. Cubillos-Angulo JM, et al. Polymorphisms in interferon pathway genes and risk of Mycobacterium tuberculosis infection in contacts of tuberculosis cases in Brazil. Int J Infect Dis. 2020;92:21-28.

50. Ma N, et al. Clinical and epidemiological characteristics of individuals resistant to $\mathrm{M}$. tuberculosis infection in a longitudinal TB household contact study in Kampala, Uganda. BMC Infect Dis. 2014;14:352.

51. Mave V, et al. Infection free "resisters" among household contacts of adult pulmonary tuberculosis. PLoS One. 2019;14(7):e0218034.

52. Stein CM, et al. Long-term stability of resistance to latent Mycobacterium tuberculosis infection in highly exposed tuberculosis household contacts in Kampala, Uganda. Clin Infect Dis. 2019;68(10):1705-1712.

53. Stein CM, et al. Resistance and susceptibility to Mycobacterium tuberculosis infection and disease in tuberculosis households in Kampala, Uganda. Am JEpidemiol. 2018;187(7):1477-1489.

54. Verrall AJ, et al. Early clearance of Mycobacterium tuberculosis: the INFECT case contact cohort study in Indonesia. J Infect Dis. 2020;221(8):1351-1360.

55. Hanifa Y, et al. Prevalence of latent tuberculosis infection among gold miners in South Africa. Int J Tuberc Lung Dis. 2009;13(1):39-46.

56. Lu LL, et al. IFN-gamma-independent immune markers of Mycobacterium tuberculosis exposure. Nat Med. 2019;25(6):977-987.

57. Seshadri C, et al. Transcriptional networks are associated with resistance to $\mathrm{Mycobac}$ terium tuberculosis infection. PLoS One. 2017;12(4):e0175844.

58. Simmons JD, et al. Monocyte metabolic programs are associated with resistance to TST/IGRA conversion. Poster presented at: Keystone Symposia, Tuberculosis: Immunity and Immune Evasion; January 16-20, 2020; Santa Fe, NM.

59. Verrall AJ, et al. Early clearance of Mycobacterium tuberculosis is associated with increased innate immune responses. J Infect Dis. 2020;221(8):1342-1350.

60. Lindestam Arlehamn CS, et al. Antigens for CD4 and CD8 T cells in tuberculosis. Cold Spring Harb Perspect Med. 2014;4(7):a018465.

61. Joosten SA, et al. Harnessing donor unrestricted T-cells for new vaccines against tuberculosis. Vaccine. 2019;37(23):3022-3030.

62. Harari A, et al. Dominant TNF- $\alpha+$ Mycobacterium tuberculosis-specific CD4+ T cell responses discriminate between latent infection and active 
disease. Nat Med. 2011;17(3):372-376.

63. Silver RF, et al. Recruitment of antigen-specific Th1-like responses to the human lung following bronchoscopic segmental challenge with purified protein derivative of Mycobacterium tuberculosis. Am J Respir Cell Mol Biol. 2003;29(1):117-123.

64 . Tsukaguchi K, et al. CD $4^{+} \alpha \beta$ T cell and $\gamma \delta$ T cell responses to Mycobacterium tuberculosis. Similarities and differences in $\mathrm{Ag}$ recognition, cytotoxic effector function, and cytokine production. JImmunol. 1995;154(4):1786-1796.

65. Ernst JD. Mechanisms of M. tuberculosis immune evasion as challenges to TB vaccine design. Cell Host Microbe. 2018;24(1):34-42.

66. Crotty S. A brief history of T cell help to B cells. Nat Rev Immunol. 2015;15(3):185-189.

67. Laidlaw BJ, et al. The multifaceted role of CD4(+) $\mathrm{T}$ cells in CD8(+) T cell memory. Nat Rev Immunol. 2016;16(2):102-111.

68. Yang JD, et al. Mycobacterium tuberculosis-specific CD4+ and CD8+ T cells differ in their capacity to recognize infected macrophages. PLoS Pathog. 2018;14(5):e1007060.

69. Tan JS, et al. Human alveolar T lymphocyte responses to Mycobacterium tuberculosis antigens: role for CD4+ and CD8+ cytotoxic T cells and relative resistance of alveolar macrophages to lysis. J Immunol. 1997;159(1):290-297.

70. Lewinsohn DA, et al. Comprehensive definition of human immunodominant CD8 antigens in tuberculosis. NPJ Vaccines. 2017;2:8.

71. VanderVen BC, et al. The minimal unit of infection: Mycobacterium tuberculosis in the macrophage. Microbiol Spectr. 2016;4(6):10.1128/ microbiolspec.TBTB2-0025-2016.

72. Weiss G, Schaible UE. Macrophage defense mechanisms against intracellular bacteria. Immunol Rev. 2015;264(1):182-203.

73. Harding CV, Boom WH. Regulation of antigen presentation by Mycobacterium tuberculosis: a role for Toll-like receptors. Nat Rev Microbiol. 2010;8(4):296-307.

74. Portal-Celhay C, et al. Mycobacterium tuberculosis EsxH inhibits ESCRT-dependent CD4 ${ }^{+}$T-cell activation. Nat Microbiol. 2016;2:16232.

75. Srivastava S, et al. Antigen export reduces antigen presentation and limits T cell control of M. tuberculosis. Cell Host Microbe. 2016;19(1):44-54.

76. Athman JJ, et al. Bacterial membrane vesicles mediate the release of Mycobacterium tuberculosis lipoglycans and lipoproteins from infected macrophages. J Immunol. 2015;195(3):1044-1053.

77. Sande OJ, et al. Mannose-capped lipoarabinomannan from Mycobacterium tuberculosis induces CD4+ T cell anergy via GRAIL. J Immunol. 2016;196(2):691-702.

78. Prados-Rosales R, et al. Mycobacteria release active membrane vesicles that modulate immune responses in a TLR2-dependent manner in mice. J Clin Invest. 2011;121(4):1471-1483.

79. Athman JJ, et al. Mycobacterium tuberculosis membrane vesicles inhibit T cell activation. J Immunol. 2017;198(5):2028-2037.

80. Achkar JM, et al. B cells and antibodies in the defense against Mycobacterium tuberculosis infection. Immunol Rev. 2015;264(1):167-181.

81. Jacobs AJ, et al. Antibodies and tuberculosis. Tuberculosis (Edinb). 2016;101:102-113.
82. Li H, Javid B. Antibodies and tuberculosis: finally coming of age? Nat Rev Immunol. 2018;18(9):591-596.

83. Tran AC, et al. Emerging themes for the role of antibodies in tuberculosis. Immune Netw. 2019;19(4):e24.

84. Kawahara JY, et al. A case for antibodies as mechanistic correlates of immunity in tuberculosis. Front Immunol. 2019;10:996.

85. Chen T, et al. Capsular glycan recognition provides antibody-mediated immunity against tuberculosis. J Clin Invest. 2020;130(4):1808-1822.

86. Li H, et al. Latently and uninfected healthcare workers exposed to TB make protective antibodies against Mycobacterium tuberculosis. Proc Natl Acad Sci U S A. 2017;114(19):5023-5028.

87. Lu LL, et al. A functional role for antibodies in tuberculosis. Cell. 2016;167(2):433-443.e14.

88. O'Shea MK, et al. Immunological correlates of mycobacterial growth inhibition describe a spectrum of tuberculosis infection. Sci Rep. 2018;8(1):14480.

89. Lu LL, et al. Antibody Fc glycosylation discriminates between latent and active tuberculosis. J Infect Dis. 2020;222(12):2093-2102.

90. Roy Chowdhury R, et al. A multi-cohort study of the immune factors associated with M. tuberculosis infection outcomes. Nature. 2018;560(7720):644-648.

91. Achkar JM, Casadevall A. Antibody-mediated immunity against tuberculosis: implications for vaccine development. Cell Host Microbe. 2013;13(3):250-262.

92. Prados-Rosales R, et al. Enhanced control of Mycobacterium tuberculosis extrapulmonary dissemination in mice by an arabinomannanprotein conjugate vaccine. PLoS Pathog. 2017;13(3):e1006250.

93. Costello AM, et al. Does antibody to mycobacterial antigens, including lipoarabinomannan, limit dissemination in childhood tuberculosis? Trans $R$ Soc Trop Med Hyg. 1992;86(6):686-692.

94. Fletcher HA, et al. T-cell activation is an immune correlate of risk in BCG vaccinated infants. Nat Commun. 2016;7:11290.

95. Kimuda SG, et al. Humoral responses to Rv1733c, Rv0081, Rv1735c, and Rv1737c DosR regulonencoded proteins of Mycobacterium tuberculosis in individuals with latent tuberculosis infection. J Immunol Res. 2017;2017:1593143.

96. Coppola M, et al. Differences in IgG responses against infection phase related Mycobacterium tuberculosis (Mtb) specific antigens in individuals exposed or not to Mtb correlate with control of TB infection and progression. Tuberculosis (Edinb). 2017;106:25-32.

97. Kunnath-Velayudhan S, et al. Dynamic antibody responses to the Mycobacterium tuberculosis proteome. Proc Natl Acad Sci U S A. 2010;107(33):14703-14708.

98. Kunnath-Velayudhan S, Gennaro ML. Immunodiagnosis of tuberculosis: a dynamic view of biomarker discovery. Clin Microbiol Rev. 2011;24(4):792-805.

99. Kunnath-Velayudhan S, et al. Proteome-scale antibody responses and outcome of Mycobacterium tuberculosis infection in nonhuman primates and in tuberculosis patients. J Infect Dis.
2012;206(5):697-705.

100.Chen T, et al. Association of human antibodies to arabinomannan with enhanced Mycobacterial opsonophagocytosis and intracellular growth reduction. J Infect Dis. 2016;214(2):300-310.

101.Song L, et al. Identification of antibody targets for tuberculosis serology using high-density nucleic acid programmable protein arrays. Mol Cell Proteomics. 2017;16(4 suppl 1):S277-S289.

102. Lee BY, Horwitz MA. Identification of macrophage and stress-induced proteins of Mycobacterium tuberculosis. JClin Invest. 1995;96(1):245-249.

103. Shah JA, et al. Nontuberculous Mycobacteria and heterologous immunity to tuberculosis. JInfect Dis. 2019;220(7):1091-1098.

104. de Valliere S, et al. Enhancement of innate and cell-mediated immunity by antimycobacterial antibodies. Infect Immun. 2005;73(10):6711-6720.

105.Zimmermann N, et al. Human isotype-dependent inhibitory antibody responses against Mycobacterium tuberculosis. EMBO Mol Med. 2016;8(11):1325-1339.

106. Choudhary A, et al. Characterization of the antigenic heterogeneity of lipoarabinomannan, the major surface glycolipid of Mycobacterium tuberculosis, and complexity of antibody specificities toward this antigen. J Immunol. 2018;200(9):3053-3066.

107. Dijkman K, et al. Prevention of tuberculosis infection and disease by local BCG in repeatedly exposed rhesus macaques. Nat Med. 2019;25(2):255-262.

108. Aguilo N, et al. Respiratory immunization with a whole cell inactivated vaccine induces functional mucosal immunoglobulins against tuberculosis in mice and non-human primates. Front Microbiol. 2020;11:1339.

109. Darrah PA, et al. Prevention of tuberculosis in macaques after intravenous BCG immunization. Nature. 2020;577(7788):95-102.

110. Chan J, et al. The role of B cells and humoral immunity in Mycobacterium tuberculosis infection. Semin Immunol. 2014;26(6):588-600.

111. Loxton AG. B cells and their regulatory functions during tuberculosis: latency and active disease. Mol Immunol. 2019;111:145-151.

112. Hertz D, et al. Increased male susceptibility to Mycobacterium tuberculosis infection is associated with smaller B cell follicles in the lungs. Sci Rep. 2020;10(1):5142.

113. Phuah J, et al. Effects of B Cell depletion on early Mycobacterium tuberculosis infection in cynomolgus macaques. Infect Immun. 2016;84(5):1301-1311.

114. Joosten SA, et al. Patients with tuberculosis have a dysfunctional circulating B-cell compartment, which normalizes following successful treatment. PLoS Pathog. 2016;12(6):e1005687.

115. Linge I, et al. B-lymphocytes forming folliclelike structures in the lung tissue of tuberculosisinfected mice: dynamics, phenotypes and functional activity. Tuberculosis (Edinb). 2017;102:16-23.

116. Maglione PJ, et al. B cells moderate inflammatory progression and enhance bacterial containment upon pulmonary challenge with Mycobacterium tuberculosis. J Immunol. 2007;178(11):7222-7234.

117. Phuah JY, et al. Activated B cells in the gran- 
ulomas of nonhuman primates infected with Mycobacterium tuberculosis. Am J Pathol. 2012;181(2):508-514.

118. Torrado E, et al. Differential and site specific impact of $\mathrm{B}$ cells in the protective immune response to Mycobacterium tuberculosis in the mouse. PLoS One. 2013;8(4):e61681.

119. Dallenga T, Schaible UE. Neutrophils in tuberculosis-first line of defence or booster of disease and targets for host-directed therapy? Pathog Dis. 2016;74(3):ftw012.

120. Moliva JI, et al. The lung mucosa environment in the elderly increases host susceptibility to Mycobacterium tuberculosis infection. J Infect Dis. 2019;220(3):514-523.

121. Patin EC, et al. Trehalose dimycolate interferes with Fc $\gamma \mathrm{R}$-mediated phagosome maturation through Mincle, SHP- 1 and Fc $\gamma$ RIIB signalling. PLoS One. 2017;12(4):e0174973.

122. Turner J, Torrelles JB. Mannose-capped lipoarabinomannan in Mycobacterium tuberculosis pathogenesis. Pathog Dis. 2018;76(4):fty026.

123. Ishikawa E, et al. Recognition of mycobacterial lipids by immune receptors. Trends Immunol. 2017;38(1):66-76.

124.Stamm CE, et al. Sensing of Mycobacterium tuberculosis and consequences to both host and bacillus. Immunol Rev. 2015;264(1):204-219.

125. Patin EC, et al. Macrophage inducible C-type lectin as a multifunctional player in immunity. Front Immunol. 2017;8:861.

126. Patin EC, et al. Mincle-mediated anti-inflammatory IL-10 response counter-regulates IL-12 in vitro. Innate Immun. 2016;22(3):181-185.

127. Russell DG, et al. Immunometabolism at the interface between macrophages and pathogens. Nat Rev Immunol. 2019;19(5):291-304.

128. Randall TD. Bronchus-associated lymphoid tissue (BALT) structure and function. $A d v$ Immunol. 2010;107:187-241.

129. Ardain A, et al. Group 3 innate lymphoid cells mediate early protective immunity against tuber- culosis. Nature. 2019;570(7762):528-532.

130. Hackett EE, et al. Mycobacterium tuberculosis limits host glycolysis and IL- $1 \beta$ by restriction of PFK-M via MicroRNA-21. Cell Rep. 2020;30(1):124-136.e4.

131. Bloom BR, Modlin RL. Mechanisms of defense against intracellular pathogens mediated by human macrophages. Microbiol Spectr. 2016;4(3):10.1128/ microbiolspec.MCHD-0006-2015.

132. Fabri $M$, et al. Vitamin $D$ is required for IFN-gamma-mediated antimicrobial activity of human macrophages. Sci Transl Med. 2011;3(104):104ra2.

133. Kimmey JM, et al. Unique role for ATG5 in neutrophil-mediated immunopathology during M. tuberculosis infection. Nature. 2015;528(7583):565-569.

134. Koeken V, et al. Trained innate immunity and resistance to Mycobacterium tuberculosis infection. Clin Microbiol Infect. 2019;25(12):1468-1472.

135. Reece ST, et al. Mycobacterium tuberculosisinfected hematopoietic stem and progenitor cells unable to express inducible nitric oxide synthase propagate tuberculosis in mice. J Infect Dis. 2018;217(10):1667-1671.

136. Tornack J, et al. Human and mouse hematopoietic stem cells are a depot for dormant Mycobacterium tuberculosis. PLoS One. 2017;12(1):e0169119.

137. Kaufmann E, et al. BCG educates hematopoietic stem cells to generate protective innate immunity against tuberculosis. Cell. 2018;172(1-2):176-190.e19.

138. Mishra BB, et al. Nitric oxide prevents a pathogen-permissive granulocytic inflammation during tuberculosis. Nat Microbiol. 2017;2:17072.

139. Yeremeev V, et al. Neutrophils exacerbate tuberculosis infection in genetically susceptible mice. Tuberculosis (Edinb). 2015;95(4):447-451.

140. Lovewell RR, et al. Granulocytes act as a niche for Mycobacterium tuberculosis growth. Mucosal Immunol. 2021;14(1):229-241.

141. Eum SY, et al. Neutrophils are the predomi- nant infected phagocytic cells in the airways of patients with active pulmonary TB. Chest. 2010;137(1):122-128.

142. Gideon HP, et al. Neutrophils express pro- and anti-inflammatory cytokines in granulomas from Mycobacterium tuberculosis-infected cynomolgus macaques. Mucosal Immunol. 2019;12(6):1370-1381.

143. Berry MP, et al. An interferon-inducible neutrophil-driven blood transcriptional signature in human tuberculosis. Nature. 2010;466(7309):973-977.

144.Prada-Medina CA, et al. Systems immunology of diabetes-tuberculosis comorbidity reveals signatures of disease complications. Sci Rep. 2017;7(1):1999.

145. Kumar NP, et al. Persistent inflammation during anti-tuberculosis treatment with diabetes comorbidity. Elife. 2019;8:e46477.

146.Blomgran R, Ernst JD. Lung neutrophils facilitate activation of naive antigen-specific $\mathrm{CD} 4+\mathrm{T}$ cells during Mycobacterium tuberculosis infection. JImmunol. 2011;186(12):7110-7119.

147. Corleis B, et al. Escape of Mycobacterium tuberculosis from oxidative killing by neutrophils. Cell Microbiol. 2012;14(7):1109-1121.

148. Dallenga T, et al. M. tuberculosis-induced necrosis of infected neutrophils promotes bacterial growth following phagocytosis by macrophages. Cell Host Microbe. 2017;22(4):519-530.e3.

149. Dallenga $\mathrm{T}$, et al. Targeting neutrophils for host-directed therapy to treat tuberculosis. Int $J$ Med Microbiol. 2018;308(1):142-147.

150. Dorhoi A, Kaufmann SH. Pathology and immune reactivity: understanding multidimensionality in pulmonary tuberculosis. Semin Immunopathol. 2016;38(2):153-166.

151. Schaible UE, et al. Strategies to improve vaccine efficacy against tuberculosis by targeting innate immunity. Front Immunol. 2017;8:1755.

152. Mayer-Barber KD, Sher A. Cytokine and lipid mediator networks in tuberculosis. Immunol Rev. 2015;264(1):264-275 